Research Paper

\title{
A complex microsatellite at chromosome 7q33 as a new prognostic marker of colorectal cancer
}

\author{
Xu Ye $\mathrm{Y}^{1,2,3}$, Hongyu Deng ${ }^{3}, \mathrm{Min}_{\mathrm{Su}}{ }^{3}$, Qianjin Liao ${ }^{3}$, Dan Huang${ }^{4}$, Duan-Fang Liao ${ }^{4}$, \\ Zhi-Qiang Xiao, ${ }^{1,2}$ and Deliang $\mathrm{CaO}^{3,4}$ \\ ${ }^{1}$ Research Center of Carcinogenesis and Targeted Therapy, Xiangya Hospital, Central South University, Changsha, Hunan \\ 410008, China \\ ${ }^{2}$ The Higher Educational Key Laboratory For Cancer Proteomics and Translational Medicine of Hunan province, Xiangya \\ Hospital, Central South University, Changsha, Hunan 410005, China \\ ${ }^{3}$ Hunan Cancer Hospital and The Affiliated Cancer Hospital of Xiangya School of Medicine, Central South University, Changsha, \\ Hunan 410013, China \\ ${ }^{4}$ Division of Stem Cell Regulation and Application, State Key Laboratory of Chinese Medicine Powder and Medicine Innovation \\ in Hunan (incubation), Hunan University of Chinese Medicine, Changsha, Hunan 410208, China \\ Correspondence to: Deliang Cao, email: dlcao1998@gmail.com \\ Zhi-Qiang Xiao, email: zqxiao2001@hotmail.com \\ Keywords: colorectal cancer, microsatellite, polymorphisms, survival, prognostic marker \\ Received: April 18,2017 Accepted: June 28, $2017 \quad$ Published: September 16, 2017 \\ Copyright: Ye et al. This is an open-access article distributed under the terms of the Creative Commons Attribution License 3.0 \\ (CC BY 3.0), which permits unrestricted use, distribution, and reproduction in any medium, provided the original author and source \\ are credited.
}

\section{ABSTRACT}

Disease-specific markers are critical for early diagnosis, targeted therapy and prognostic prediction of diseases. Current study reports a complex microsatellite as a new prognostic marker of sporadic colorectal cancer. This microsatellite located at Chromosome 7q33 is composed of three tetranucleotide tandem repeats, $(\text { TTCC })_{2}(\text { TCCC })_{5}(\text { TCCT })_{7}$, flanked by a CT-rich sequence. We analyzed polymorphisms of this microsatellite in 158 sporadic colorectal cancer, 143 matched normal adjacent tissues (NAT) and 150 health donors. Our results showed that this complex microsatellite was instable with polymorphic frequency of $77.2 \%$ in colorectal cancer, $52.4 \%$ in NAT and $54.7 \%$ in health donors $(p<0.01)$ when compared to reference sequence. In the three tandem repeats, (TCCT), site was most polymorphic accounting for over $\mathbf{7 0 . 0 \%}$ of polymorphisms in this complex microsatellite, followed by (TTCC) site for approximately $20 \%$. Polymorphisms in (TCCC) $)_{5}$ was rare. Polymorphisms at the (TCCT) $)_{7}$ site were mainly insertions of 1 to 4 copies of TCCT $(88.6 \%)$, and deletions occurred in about $6.4 \%$ of cases. The (TTCC) ${ }_{2}$ site was featured with one copy TTCC insertions. Pair-wise analyses between colorectal tumors and NAT revealed that 88 of $121(72.7 \%)$ tumors displayed expansion, contraction or both in these tetranucleotide tandem repeats when compared to NAT. A cross-analysis with clinicopathological data of 158 colorectal cancers revealed that polymorphic alterations of the microsatellite associated with less lymphatic metastasis $(p<0.001)$, and the colorectal cancer patients with polymorphic changes in this microsatellite demonstrated better survival $(n=112, p=0.0058)$. Together these data suggest that this complex microsatellite is a potential prognostic marker of sporadic colorectal cancer.

\section{INTRODUCTION}

Colorectal cancer (CRC) is a leading cause of cancer-related deaths in the United States; approximately 1 in 19 Americans (5.29\%) will suffer from colorectal cancer during their life-time [1]. Individuals who have inflammatory bowel disease (IBD), i.e., ulcerative colitis and Crohn's disease, have high risk of developing colorectal cancer, which increases exponentially with duration of the disease; surveillance of colorectal cancer 
in IBD patients has important clinical impact in prevention of IBD-associated cancer death [2, 3].

Advanced colorectal cancer has a high mortality rate; and early diagnosis is the only practical way to improve the disease-free survival of cancer patients [4-6]. Nearly $75 \%$ of colorectal cancer develops among people free of IBD and a familial history of hereditary non-polyposis colorectal cancer (HNPCC), colorectal neoplasia, or familial adenomatous polyposis (FAP), so called sporadic colorectal cancer. This makes the early diagnosis difficult due to lack of screening [7-10]. Up to date, colonoscopy screening in populations of IBD, FAP and HNPCC or subjects over 50 years old is an effective procedure for the surveillance and early diagnosis of colorectal cancer and has reduced the mortality in past decades [11-13]. However, colonoscopy is invasive and uncomfortable with potential complications, such as postpolypectomy, postbiopsy bleeding, puncturing of the colon lining and postpolypectomy syndrome [14 16]. Colonoscopy is also costly and requires sedation and thorough preparation of the colon, which often results in reduced patient compliance. Fecal occult blood test (FOBT) is non-invasive and home-based and newer methods, such as Hemoccult SENSA and fecal immunochemical tests (FIT), have improved sensitivity; however, their specificity is limited, often leading to false diagnosis [17-19]. In addition, FOBT fails to detect non-bleeding polyps and has poor specificity for colorectal cancer and adenoma [17, 20]. Hence, it is needed to identify and validate non-invasive, affordable, highly sensitive and specific biomarkers for screening and early diagnosis and/or prognostic prediction of colorectal cancer to improve the clinical management of this lethal disease.

Microsatellites have been shown to be potent genetic markers of cancers. Microsatellite instability (MSI) often correlates with certain age-related diseases, including colorectal cancer [21-24]. Microsatellites are defined as 1 to 6 repetitive DNA sequences distributed throughout the genome $[25,26]$. MSI is a form of genomic instability that results from molecular defects in DNA mismatch repair machinery, causing frameshift mutations in highly repetitive microsatellite sequences [21, 23, 27]. In hereditary non-polyposis colon cancer (HNPCC), a disease caused by the mutations of DNA mismatch repair genes $h M S H 2$ or $h M L H 1$, MSI occurs in up to $90 \%$ of cases, compared to approximately $15 \%$ in sporadic tumors $[21,28]$. A high level of MSI in colorectal cancer usually indicates a favorable response to adjuvant chemotherapy and better prognosis [25, 29].

This study found that a microsatellite located at Chromosome 7q33 functions as a prognostic marker of colorectal cancer. It is a complex microsatellite composed of three tetranucleotide tandem repeats, (TTCC) ${ }_{2}(\text { TCCC })_{5}(\text { TCCT })_{7}$, flanked by CT-rich sequences. Our results demonstrated that this complex microsatellite is instable and that its polymorphic alterations were associated with less lymph node metastasis of colorectal cancer and better patient survival.

\section{RESULTS}

\section{Identification of a complex repetitive sequence at Chromosome $7 q 33$}

DNA sequence analysis of the AKR1B10 promoter region, a gene frequently silenced in inflammatory bowel disease and colorectal cancer [31,32], revealed a repetitive sequence composed of tandem 4-nucleotide repeats, $(\mathrm{TTCC})_{2}(\mathrm{TCCC})_{5}(\mathrm{TCCT})_{7}$, with a flanking $\mathrm{C}$ and $\mathrm{T}$ enriched sequence (Figure 1A). This repetitive sequence is located at Chromosome 7 q33 (NC 000007.14, nucleotide number 134526936 - 134527095) (Figure 1B). In this study, we used this sequence as a reference sequence.

\section{Polymorphisms of this complex microsatellite in colorectal cancer}

To characterize this repetitive sequence, we evaluated its polymorphisms and possible clinical implications in colorectal cancer. We first assessed its polymorphic changes in healthy subjects using the DNA sequence of $\mathrm{NC} 000007.14$ as a reference sequence. Genomic DNA was amplified by PCR for conventional sequencing analysis. Figure 2 shows the chromatography of the sequencing data and representatives of polymorphic changes. Our results showed that this CT-enriched repetitive sequence is instable with polymorphic variations in 82 of $150(54.7 \%)$ healthy donor subjects when compared to the reference sequence. We further analyzed the variant patterns and location in this site. The results showed that polymorphic variations mainly occurred in the tetranucleotide tandem repeats (TCCT) and (TTCC) $)_{2}$. The former accounted for $73.8 \%$ of overall polymorphic changes in this complex microsatellite and the latter denoted $24.3 \%$ of variations. The polymorphisms in the (TCCC) $)_{5}$ site were rare accounting only for $1.9 \%$ of overall polymorphisms (Table 1). As for the polymorphic patterns, the (TTCC) $)_{2}$ site was featured with insertions of a single copy TTCC, but the (TCCT) ${ }_{7}$ exhibited insertions of 1 - 4 copies of TCCT as main polymorphic forms. A single copy deletion of TCCT was detected in $14.6 \%$ of cases. A rare variant of a single copy TCTT insertion was noted in $2.9 \%$ of cases (Table 1 ). Figure $2 \mathrm{~B} \& 2 \mathrm{C}$ demonstrates the representative chromatography of single copy TTCC and TCCT insertions in healthy donors. Figure $3 \mathrm{~A}$ shows the representative types of polymorphisms in healthy donors.

We further analyzed the polymorphisms of this microsatellite in 158 colorectal cancer and 143 normal adjacent colorectal tissues (NAT). Results showed that in the NAT samples, polymorphic frequency, types and location in this complex microsatellite were pretty similar to those in the healthy donors. In the colorectal cancer tissues, the variant types and spectra were also similar to those in NAT and healthy donors, but when compared to reference sequence, the polymorphic frequency was higher at $77.2 \%$ in tumors vs. $52.4 \%$ in NAT $(p<0.01)$. Figure $3 B$ 


\section{A) Sequence of complex microsatellite}

1 TTGTTTTTTC TTCCTTCTTT GCTTTGCTTT СTTTCTCTCC TTCCTTCTTT CTITCCттCC

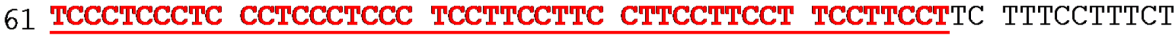

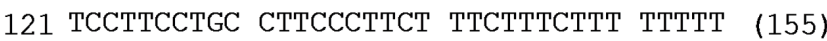

Complex Microsatellite: (TTCC $)_{2}(\mathrm{TCCC})_{5}(\mathrm{TCCT})_{7}(-604$ to $-549 \mathrm{bp})$

\section{B) Chromosomal location}

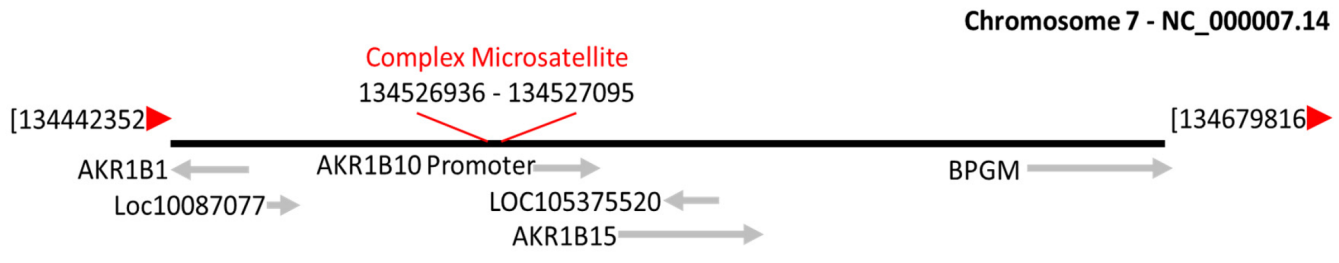

Figure 1: A complex microsatellite at Chromosome 7q33. (A) Sequence and components of this microsatellite, consisting of three consecutive tetranucleotide tandem repeats flanked by a $\mathrm{C}$ and $\mathrm{T}$ - enriched sequence. (B) Diagram of the chromosomal location of this microsatellite.

\section{A) Chromatography: $(\text { TTCC })_{2}(\text { TCCC })_{5}(\text { TCCT })_{7}$}

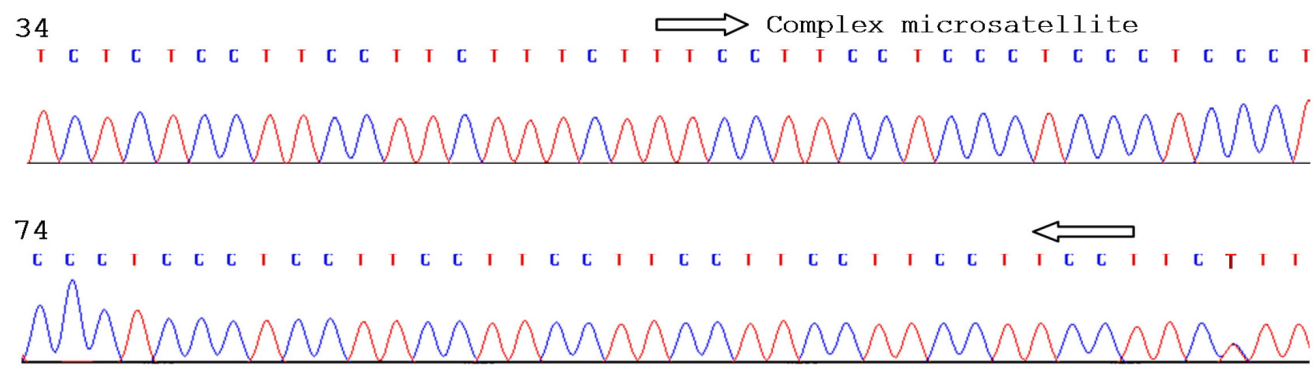

B) TTCC insertion

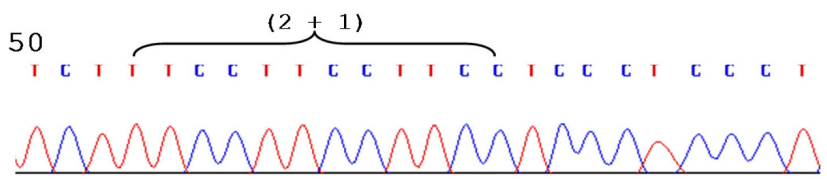

C) TCCT insertion

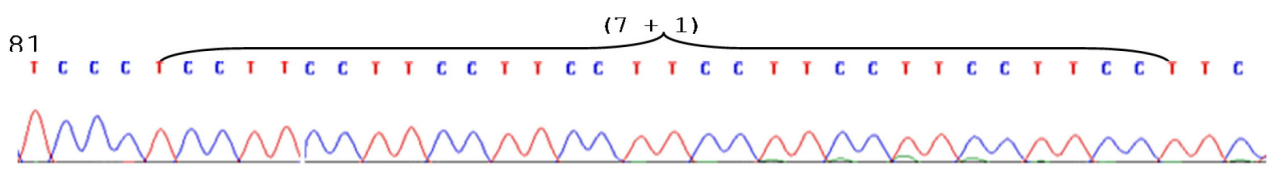

Figure 2: Chromatography of this microsatellite and representative polymorphisms. (A) Sequencing chromatography of this microsatellite without polymorphisms, showing quality of the DNA sequencing data. (B) A sequence chromatography showing a single copy TTCC insertion. (C) A sequence chromatography showing a single copy TCCT insertion. 
Table 1: Microsatellite polymorphisms compared to reference sequence

\begin{tabular}{|c|c|c|c|}
\hline & $\begin{array}{c}\text { Donors } \\
(n=150)\end{array}$ & $\begin{array}{c}\text { NAT } \\
(n=143)\end{array}$ & $\begin{array}{c}\text { CRC } \\
(n=158)\end{array}$ \\
\hline \multicolumn{4}{|l|}{ Polymorphic frequency } \\
\hline Polymorphism (\%) ${ }^{1}$ & $82(54.7)$ & $75(52.4)$ & $122(77.2)$ \\
\hline Non-polymorphism (\%) & $68(45.3)$ & $68(47.6)$ & $36(22.8)$ \\
\hline \multicolumn{4}{|l|}{ Types and rates } \\
\hline \multicolumn{4}{|l|}{ TTCC site } \\
\hline TTCC insertions $(\%)^{2}$ & $25(24.3)$ & $23(23.5)$ & $36(20.9)$ \\
\hline \multicolumn{4}{|l|}{ TCCC site } \\
\hline TCCC deletions $(\%)$ & $2(1.9)$ & $1(1.0)$ & $8(4.7)$ \\
\hline TCCC insertion (\%) & 0 & $1(1.0)$ & $5(2.9)$ \\
\hline Subtotal (\%) & $2(1.9)$ & $2(2.0)$ & $13(7.6)$ \\
\hline \multicolumn{4}{|l|}{ TCCT site } \\
\hline TCCT deletions $(\%)$ & $15(14.6)$ & $12(12.2)$ & $11(6.4)$ \\
\hline \multicolumn{4}{|l|}{ TCCT insertions (\%) } \\
\hline $7+1(\%)$ & $15(14.6)$ & $14(14.3)$ & $34(19.8)$ \\
\hline $7+2(\%)$ & $31(30.1)$ & $25(25.5)$ & $48(27.9)$ \\
\hline $7+3(\%)$ & $8(7.8)$ & $11(11.2)$ & $22(12.8)$ \\
\hline $7+4(\%)$ & $4(3.9)$ & $9(9.2)$ & $5(2.9)$ \\
\hline TCTT insertions $(\%)$ & $3(2.9)$ & $2(2.0)$ & $3(1.7)$ \\
\hline Subtotal $(\%)^{3}$ & $76(73.8)$ & $73(74.5)$ & $123(71.5)$ \\
\hline Total $(\mathrm{PF} / \mathrm{V})^{4}$ & $103(1.26)$ & $98(1.31)$ & $172(1.41)$ \\
\hline
\end{tabular}

CRC, colorectal cancer; NAT, normal adjacent tissues

${ }^{1}$ Subjects (percentage) with polymorphisms compared to reference sequence. $\mathrm{p}<0.01$, compared to NAT and donors.

${ }^{2} \mathrm{p}<0.05$, compared to TCCC site. $\%$ in parenthesis indicates percentage in overall variations.

${ }^{3} \mathrm{p}<0.01$, compared to TTCC and TCCC sites.

${ }^{4} \mathrm{PF} / \mathrm{V}$, polymorphic frequency per site; more than one type of polymorphisms in some sites.

\& 3C shows the representative types of polymorphisms in colorectal cancer and normal adjacent tissues. The polymorphic types and rates of this complex microsatellite in colorectal cancer and normal adjacent tissues are summarized in Table 1. These data suggest the instability of this complex microsatellite in carcinogenic progression.

To further address this question, we performed a pair-wise analysis of the polymorphic alterations in this microsatellite between the tumor tissues and matched NAT. In this study, a total of 121 pairs of colorectal specimens were collected and analyzed, and compared to the NAT, $88(72.7 \%)$ of tumors displayed expansions, contractions or both in this complex microsatellite, suggesting that rapid cell proliferation and DNA replication during cancer development may stimulate polymorphic alterations. Figure 4 shows representative polymorphic variations in tumors in comparison with NAT samples.

\section{Negative association of the complex microsatellite polymorphisms with lymphatic metastasis of colorectal cancer}

In view of the high polymorphic variations, we further assessed the clinical significance of this complex microsatellite. A cross-analysis of the polymorphisms with clinicopathological parameters collected from 158 
colorectal cancer was performed. As demonstrated in Table 2, tumors with polymorphic alterations had less lymph node metastasis $(\mathrm{p}<0.001)$ compared to those with no polymorphic changes, suggesting its potential as a prognostic marker. Polymorphisms of this microsatellite had no correlation with patient age, sex or tumor size.

\section{Better disease-free survival of patients with polymorphisms of this complex microsatellite}

With the finding of negative indication in lymph node metastasis, we further observed the correlation of the microsatellite instability with patient survival. We followed up the colorectal cancer patients subjected to the polymorphic analysis of this complex microsatellite, and survival data were obtained in 112 patients. Kaplan-Meier plots showed that patients with polymorphic variations of this complex microsatellite had significantly better disease-free survival $(n=112, p=0.0058)$ (Figure 5). This data suggests that instability of this complex microsatellite may be a prognostic marker of colorectal cancer.

\section{DISCUSSION}

Colorectal cancer is a serious public health issue. Through decades of efforts, colorectal cancer biomarkers, including microsatellites, have contributed to the diagnosis and prognosis, as well as prediction of treatment response. In this study, we identified and characterized a novel microsatellite marker that is located at Chromosome 7q33.

This microsatellite is composed of three tetranucleotide tandem repeats, $(\text { TTCC })_{2}(\text { TCCC })_{5}(\text { TCCT })_{7}$. Of the three different repeats, the (TCCT) ${ }_{7}$ site was most instable and accounted for majority of the polymorphic variations, followed by the (TTCC), while the (TCCC) site is virtually stable with very rare variations. Polymorphisms of microsatellites in tumors are often featured with expansions (insertions) and/or contractions (deletions) (27). Similarly, polymorphic type analyses

A) DNA from normal donors

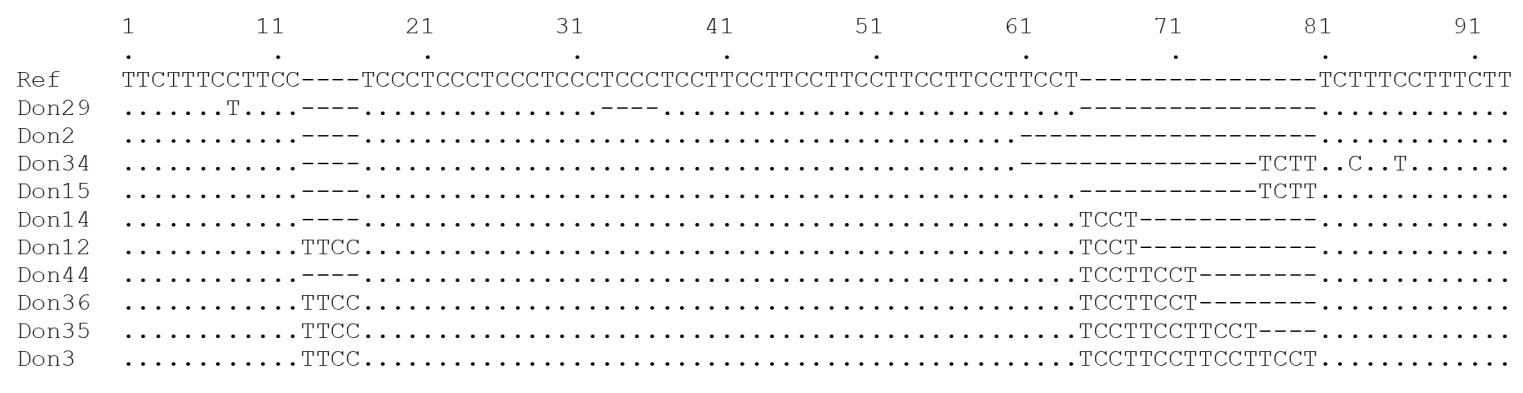

B) Colorectal cancer

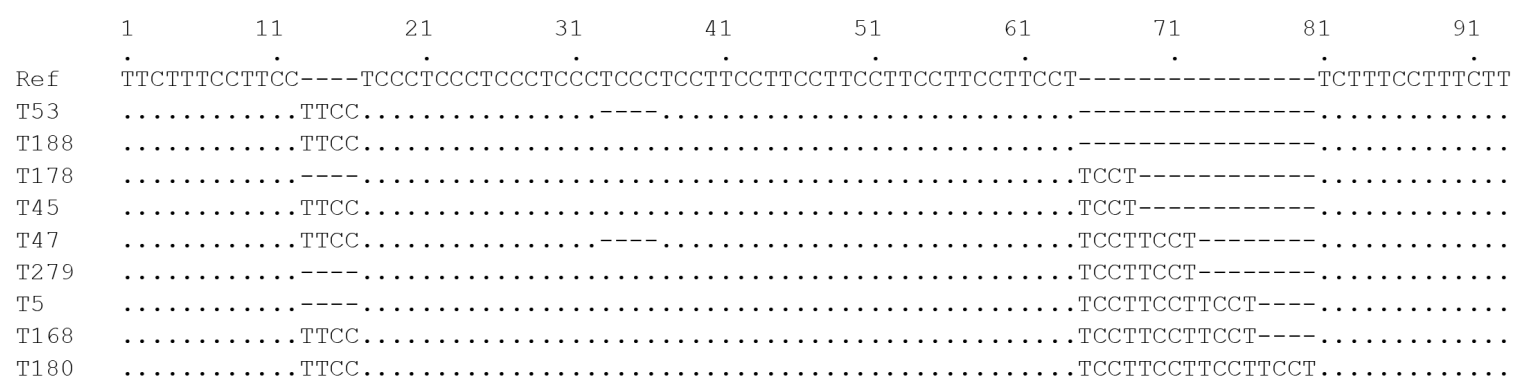

C) Normal adjacent tissues

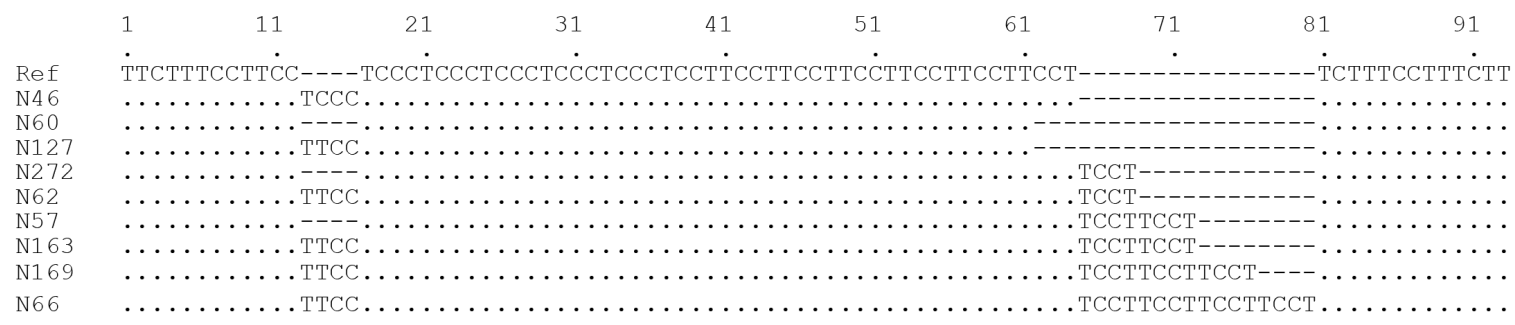

Figure 3: Polymorphic types of the complex microsatellite. Presented sequences represent different types of polymorphic alterations observed in healthy donors (A), colorectal cancer (B) and normal adjacent tissues (C). 
showed that expansions or contractions were detected and that in some cases, expansions and contractions both occurred. However, frequency of the expansions or contractions varied. In the (TCCT) $)_{7}$ site, TCCT insertions, varied from one to four copies, were the main polymorphic forms and deletions with one copy of TCCT were a less event. Polymorphisms at the (TTCC) site were less frequent and featured with insertions of a single copy of TTCC. Mutations and/or epi-mutations in genes of DNA mismatch repair system (MMR), such as MLH1, MSH2, MSH6 and PMS2, leads to alterations in repeated DNA sequences (i.e., microsatellites), so-called microsatellite instability (MSI) [33]. However, why these tetranucleotide repeats demonstrated differential frequency of polymorphisms is currently unclear.

Comparative analyses between healthy donors, colorectal cancers and adjacent normal tissues demonstrated that polymorphisms occurred in all three cohorts of subjects when compared to the reference sequence. In these specimens, the polymorphic types were similar, but the polymorphic frequency was significantly higher in the tumor tissues than in NAT and healthy donors $(\mathrm{p}<0.01)$, indicating microsatellite instability during the tumorigenesis. This was further confirmed by paired analyses between colorectal cancers and matched normal adjacent tissues. Data showed that

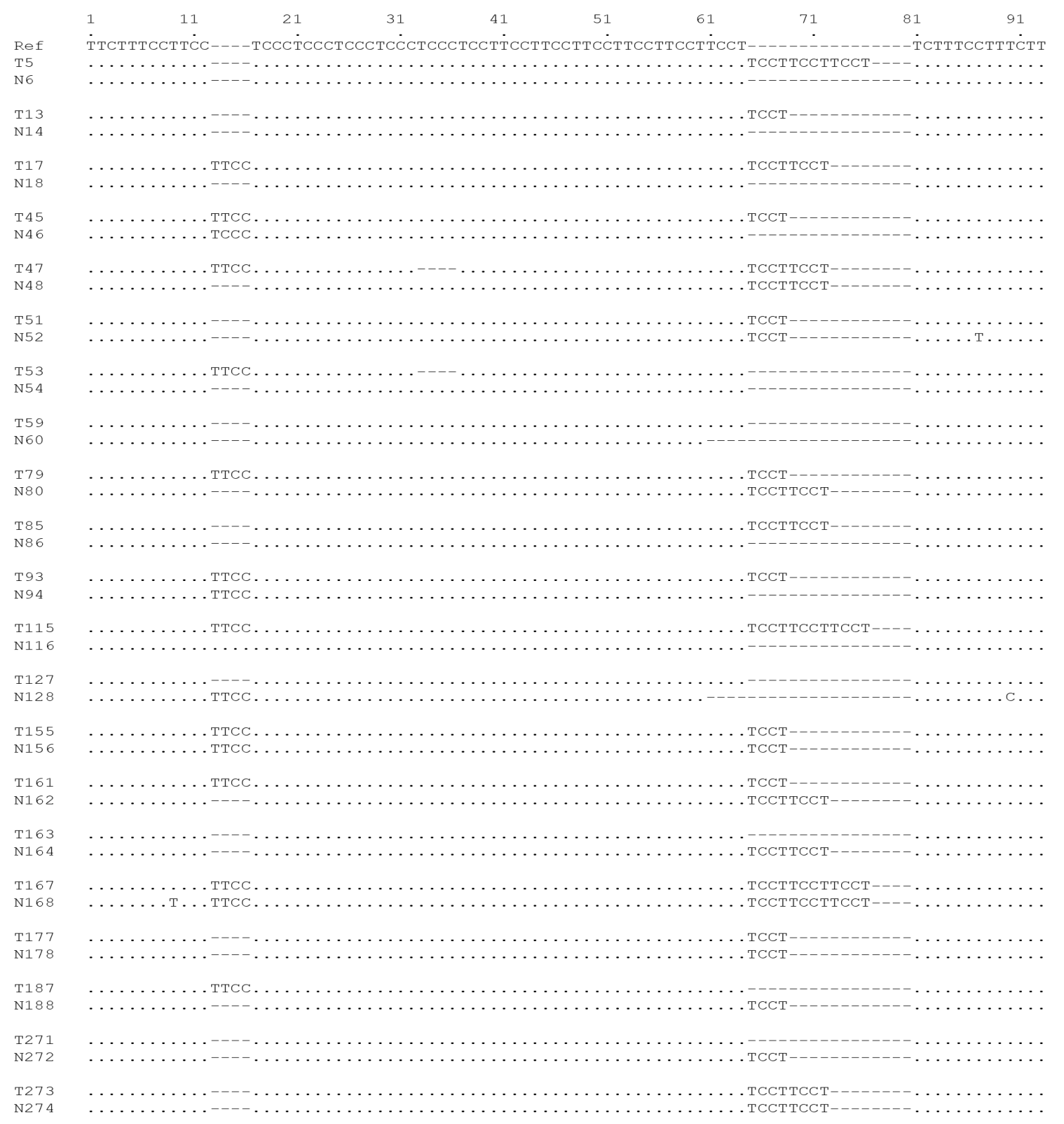

Figure 4: Polymorphic variations in sporadic colorectal cancer. DNA sequences of this complex microsatellite from paired colorectal cancer and normal adjacent specimens were subjected to paired polymorphic analyses. Data show that expansions, contractions or both that occurred in some tumors during tumorigenesis. 
Table 2: Correlation between microsatellite polymorphisms and clinicopathological parameters in colorectal cancer

\begin{tabular}{lccc}
\hline & & Colorectal Cancer $(\boldsymbol{n}=\mathbf{1 5 8})$ & \\
\cline { 2 - 4 } & Polymorphisms & Non-polymorphisms & P-Value \\
\hline Subtotal & $122(77.2)$ & $36(22.8)$ & 0.8508 \\
Age (yr) & & & \\
Mean & 60.8 & 61.7 & \\
Median & 65.0 & 63.0 & 0.3653 \\
Range & $23.0-89.0$ & $45.0-84.0$ & \\
Sex & & & \\
Male & 65 & 20 & \\
Female & 57 & & \\
Tumor size (cm) & & 6.16008 \\
Mean & 5.7 & 4.5 & \\
Median & 4.2 & $3.5-10.3$ & \\
Range & $2.3-11.8$ & $27(75.0 \%)$ & \\
Lymph node metastasis & & $9(25.0 \%)$ & \\
Positive & $35(28.7 \%)$ & $(71.3 \%)$ & \\
Negative & &
\end{tabular}

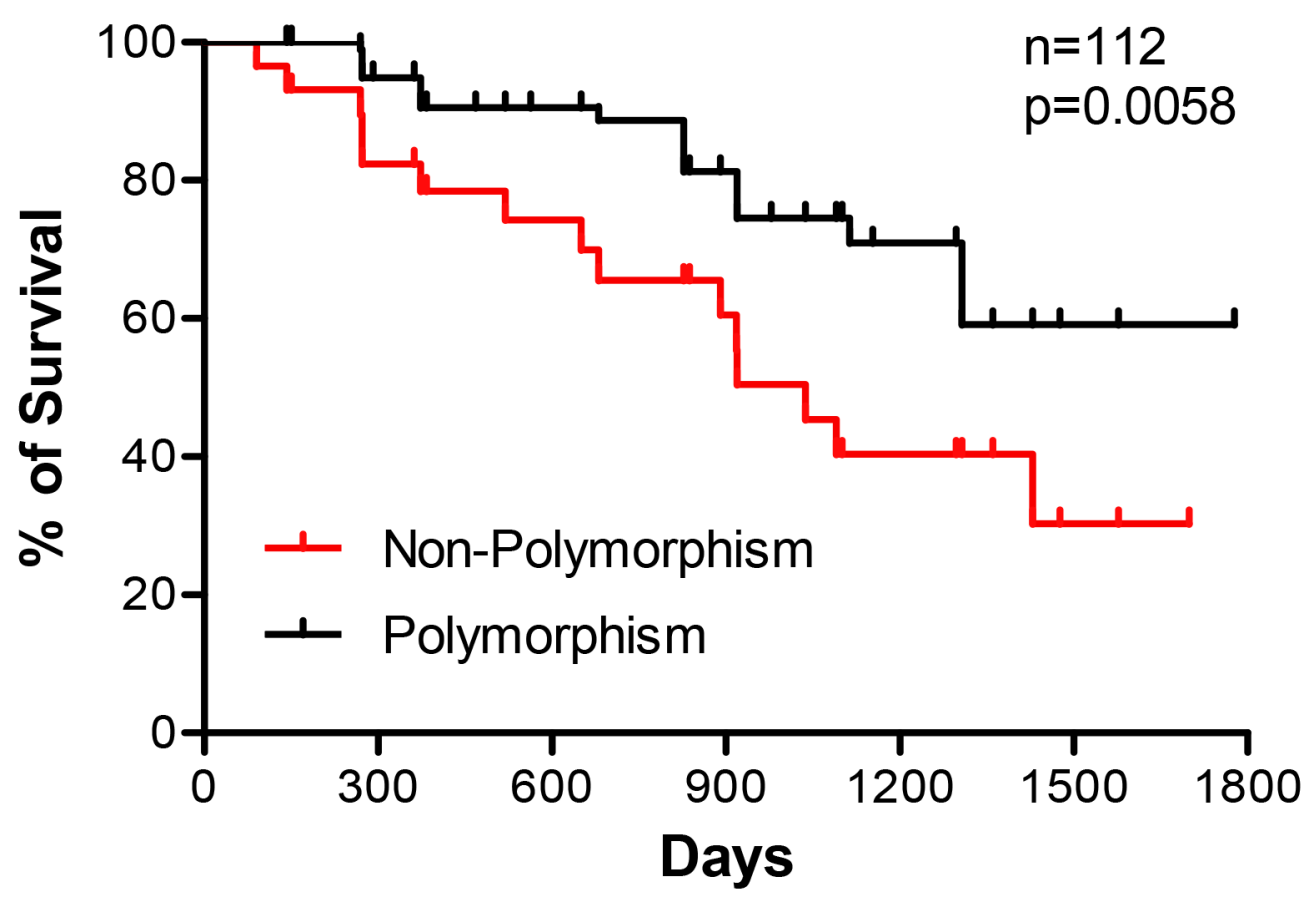

Figure 5: Correlation of microsatellite polymorphisms with disease-free survival of colorectal cancer patients. KaplanMeier plots of patient survival were used for survival analysis. Data show a better survival of patients with polymorphic variations compared to those with no changes $(\mathrm{n}=112, \mathrm{p}=0.0058)$. 
$72.7 \%$ of tumors displayed insertions, deletions or both in this complex microsatellite when compared to matching normal adjacent tissues. These data suggests that rapid cell proliferation and DNA replication during cancer development may push polymorphic alterations of this microsatellite.

Classic microsatellite markers used clinically are monocleotide and dinucleotide repeats, and their MSI occurs in approximately $15 \%$ of sporadic colorectal cancer [33]. However, a specific type of MSI, named elevated microsatellite alterations at selected tetranucleotide repeats (EMAST), may occur in up to $60 \%$ of sporadic colorectal cancer [34]. This complex microsatellite identified here is composed of three tetranucleotide repeats, $(\text { TTCC })_{2}(\text { TCCC })_{5}(\text { TCCT })_{7}$, which may explain the high polymorphic frequency of $72.7 \%$ in total of three sites in tumors when compared to normal adjacent tissues. This complex microsatellite may be a new complex EMAST.

This study also revealed that the polymorphisms of this complex microsatellite associated negatively with local lymph node metastasis, a critical factor for patient prognosis. This observation is consistent with literature reports that colorectal tumors with high MSI levels had less metastasis and better prognosis. Thus the patients with high MSI tumors have a higher disease-free survival rate compared to those without MSI [29, 35-37]. Indeed, a survival analysis in this study exhibited that the patients with polymorphic variations in this microsatellite had better disease-free survival, suggesting that this complex microsatellite may be a new positive prognostic marker of colorectal cancer. We have not a clear answer yet for decreased metastasis of the tumors with MSI, but the favorable prognosis may be ascribed to the better response to DNA damage agent 5-fluorouracil, a first-line drug for colorectal cancer.

Stool DNA has become a useful diagnostic specimen for disease screening and diagnosis. For instance, stool DNA kit (PreGen-Plus, MA) is developed to detect a panel of DNA markers involved in the colorectal cancer [38, 39]. It may be of potential value of early diagnosis or prognosis prediction by using the developed stool DNA techniques to exam the polymorphic changes in this complex microsatellite. The presence of a new polymorphism in this microsatellite in the stool DNA may indicate the presence of a tumor mass or a better prognosis for patients with developed colorectal cancer.

In summary, we identified and characterized a complex microsatellite that is located at Chromosome 7q33. This microsatellite has two polymorphic hot spots, $(\mathrm{TCCT})_{7}$ and (TTCC) $)_{2}$, and polymorphic alterations of these spots positively associated with survival of the colorectal cancer, being a potential prognostic marker of this disease. A large cohort of prospective study may be merited to define the prognostic value of this microsatellite in colorectal cancer and the specificity and sensitivity of this complex microsatellite as a prognostic marker.

\section{MATERIALS AND METHODS}

\section{Ethics statement}

IRB protocols were approved by Hunan Cancer Hospital Committee for Research Involving Human subjects.

\section{Human sample procurement}

With approved IRB protocols, we procured a total of 158 sporadic colorectal cancer and 143 normal adjacent colon specimens in this study, which were surgically resected from Hunan Cancer Hospital. All specimens were quality-controlled by pathologists. Clinicopathological data, including patient age and sex, and tumor size, differentiation, and lymph node metastasis were collected. Normal adult genomic DNA was extracted from blood of healthy donors. Registered patients were followed up for 5 years of disease-free survival.

\section{DNA extraction}

Genomic DNA from surgical specimens was extracted as previously described [30]. After being completely dissolved in TE buffer $(10 \mathrm{mM}$ Tris. $\mathrm{Cl}$ and $1 \mathrm{mM}$ EDTA, pH 8.0), DNA was quantitated at $260 \mathrm{~nm}$ using a spectrophotometer (Beckman, CA).

\section{PCR and DNA sequencing}

Genomic DNA (200 ng) were used for PCR amplification at $95^{\circ} \mathrm{C}$ for $45 \mathrm{sec}, 60^{\circ} \mathrm{C}$ for $30 \mathrm{sec}$, and $72^{\circ} \mathrm{C}$ for $90 \mathrm{sec}$ for 30 cycles, after an initial denature at $95^{\circ} \mathrm{C}$ for $5 \mathrm{~min}$. Forward primer was $5^{\prime}$ GAA GTA TAA GAT TTT TCA CTC ATA G, and reverse primer was 5'GAA AGG AGA ATC ACT TGA ACC T GGG. PCR products were purified with PCR DNA purification kit (Qiagen, CA) and the sequences were read with a nested primer (5'CAT GCC AAT TGC CTT CTA TG) by a commercial company.

\section{Alignment of DNA sequences and data analysis}

Sequence chromatograms were visualized and edited using Sequencher v4.6 (Gene Codes Corporation, MI) (24). Edited DNA sequences were saved as text files and contigs containing all sequences for a particular group. Pair-wise comparisons were assembled and exported as a NEXUS file. The 5 ' end of the microsatellite was marked by a TTCT motif for alignment. Gaps were inserted to indicate the insertions or deletions. The resulting alignments were edited using MacClade v4.06 (Sinauer Associates, Inc., MA) and analyzed for polymorphic changes. The microsatellite and flanking sequences in the database of the National Center for Biotechnology Information were used as a reference. 


\section{Statistical analysis}

Chi-square tests of independence, or Fisher's exact tests when appropriate, were used to examine the relationship of microsatellite polymorphisms with tissue type, as well as with the clinical parameters of the cancer, such as gender, tumor differentiation, and lymph node metastasis. Analysis of variance was used to compare the polymorphism rates between sites. Independent group $t$-tests were used to assess the relationship of polymorphisms with patient age and tumor size. Patient survival was plotted by Kaplan-Meier analysis. Results were considered statistically significant with $p<0.05$. All analyses were performed using SAS v9.1 software, (SAS Institute Inc., NC).

\section{Author contributions}

$\mathrm{XY}$ run majority of experiments, patient recruitment, data analysis and draft of the manuscript; HD assisted with experimental preparation and wet bench work; MS, QL and $\mathrm{DH}$ participated in the data collection and analysis; DFL aided in research design and data interpretation; DC and ZQX served in the research design, data analysis and interpretation and finalized the manuscript.

\section{CONFLICTS OF INTEREST}

Authors declare no conflicts of interest.

\section{FUNDING}

This work was supported in part by National Natural Science Foundation of China (81472465 to DC), National Basic Research Program of China (2013CB910502; to ZQX) and the Natural Science Foundation of Guangxi Provence (2015GXNSFEA139003 to DFL).

\section{REFERENCES}

1. Siegel RL, Miller KD, Fedewa SA, Ahnen DJ, Meester RG, Barzi A, Jemal A. Colorectal cancer statistics, 2017. CA Cancer J Clin. 2017; 67:177-193.

2. Danese S, Malesci A, Vetrano S. Colitis-associated cancer: the dark side of inflammatory bowel disease. Gut. 2011; 60:1609-1610.

3. Rogler G. Chronic ulcerative colitis and colorectal cancer. Cancer letters. 2014; 345:235-241.

4. Kuipers EJ, Grady WM, Lieberman D, Seufferlein T, Sung JJ, Boelens PG, van de Velde CJ, Watanabe T. Colorectal cancer. Nature reviews Disease primers. 2015; 1:15065.

5. Marley AR, Nan H. Epidemiology of colorectal cancer. International journal of molecular epidemiology and genetics. 2016; 7:105-114.
6. Vendrell E, Morales C, Risques RA, Capella G, Peinado MA. Genomic determinants of prognosis in colorectal cancer. Cancer letters. 2005; 221:1-9.

7. Winawer SJ, Schottenfeld D, Flehinger BJ. Colorectal cancer screening. Journal of the National Cancer Institute. 1991; 83:243-253.

8. Kolligs FT. Diagnostics and Epidemiology of Colorectal Cancer. Visceral medicine. 2016; 32:158-164.

9. Pavlovic-Calic N, Muminhodzic K, Zildzic M, Smajic M, Gegic A, Alibegovic E, Salkic N, Jovanovic P, Basic $\mathrm{M}$, Iljazovic S. Genetics, clinical manifestations and management of FAP and HNPCC. Medicinski arhiv. 2007; 61:256-259.

10. Pistorius S, Gorgens H, Plaschke J, Hoehl R, Kruger S, Engel C, Saeger HD, Schackert HK. Genomic rearrangements in MSH2, MLH1 or MSH6 are rare in HNPCC patients carrying point mutations. Cancer letters. 2007; 248:89-95.

11. Lynch PM. Prevention of colorectal cancer in highrisk populations: the increasing role for endoscopy and chemoprevention in FAP and HNPCC. Digestion. 2007; 76:68-76.

12. Kumar AS, Lee JK. Colonoscopy: Advanced and Emerging Techniques-A Review of Colonoscopic Approaches to Colorectal Conditions. Clinics in colon and rectal surgery. 2017; 30:136-144.

13. Hazewinkel Y, Dekker E. Colonoscopy: basic principles and novel techniques. Nature reviews Gastroenterology \& hepatology. 2011; 8:554-564.

14. Levin TR, Zhao W, Conell C, Seeff LC, Manninen DL, Shapiro JA, Schulman J. Complications of colonoscopy in an integrated health care delivery system. Annals of internal medicine. 2006; 145:880-886.

15. Dafnis G, Ekbom A, Pahlman L, Blomqvist $P$. Complications of diagnostic and therapeutic colonoscopy within a defined population in Sweden. Gastrointestinal endoscopy. 2001; 54:302-309.

16. Day LW, Kwon A, Inadomi JM, Walter LC, Somsouk M. Adverse events in older patients undergoing colonoscopy: a systematic review and meta-analysis. Gastrointestinal endoscopy. 2011; 74:885-896.

17. Anderson WF, Guyton KZ, Hiatt RA, Vernon SW, Levin B, Hawk E. Colorectal cancer screening for persons at average risk. Journal of the National Cancer Institute. 2002; 94:1126-1133.

18. Swaroop VS, Larson MV. Colonoscopy as a screening test for colorectal cancer in average-risk individuals. Mayo Clinic proceedings. 2002; 77:951-956.

19. Rennert G, Kislitsin D, Brenner DE, Rennert HS, Lev Z. Detecting K-ras mutations in stool from fecal occult blood test cards in multiphasic screening for colorectal cancer. Cancer letters. 2007; 253:258-264. 
20. Potter MB. Strategies and resources to address colorectal cancer screening rates and disparities in the United States and globally. Annual review of public health. 2013; 34:413-429.

21. Grady WM. Genomic instability and colon cancer. Cancer metastasis reviews. 2004; 23:11-27.

22. Fleisher AS, Esteller M, Harpaz N, Leytin A, Rashid A, Xu Y, Liang J, Stine OC, Yin J, Zou TT, Abraham JM, Kong D, Wilson KT, et al. Microsatellite instability in inflammatory bowel disease-associated neoplastic lesions is associated with hypermethylation and diminished expression of the DNA mismatch repair gene, hMLH1. Cancer research. 2000; 60:4864-4868.

23. Woerner SM, Kloor M, Mueller A, Rueschoff J, Friedrichs N, Buettner R, Buzello M, Kienle P, Knaebel HP, Kunstmann E, Pagenstecher C, Schackert HK, Moslein $\mathrm{G}$, et al. Microsatellite instability of selective target genes in HNPCC-associated colon adenomas. Oncogene. 2005; 24:2525-2535.

24. Notarnicola M, Gristina R, Messa C, Cariola F, Fiorente P, Caruso ML, Gentile M, Di Leo A. Oestrogen receptors and microsatellite instability in colorectal carcinoma patients. Cancer letters. 2001; 168:65-70.

25. Shankaran V, Wisinski KB, Mulcahy MF, Benson AB $3 \mathrm{rd}$. The role of molecular markers in predicting response to therapy in patients with colorectal cancer. Molecular diagnosis \& therapy. 2008; 12:87-98.

26. Guleng G, Lovig T, Meling GI, Andersen SN, Rognum TO. Mitochondrial microsatellite instability in colorectal carcinomas--frequency and association with nuclear microsatellite instability. Cancer letters. 2005; 219:97-103.

27. Ghimenti C, Tannergard P, Wahlberg S, Liu T, Giulianotti PG, Mosca F, Fornaciari G, Bevilacqua G, Lindblom A, Caligo MA. Microsatellite instability and mismatch repair gene inactivation in sporadic pancreatic and colon tumours. British journal of cancer. 1999; 80:11-16.

28. Chialina SG, Fornes C, Landi C, de la Vega Elena CD, Nicolorich MV, Dourisboure RJ, Solano A, Solis EA. Microsatellite instability analysis in hereditary nonpolyposis colon cancer using the Bethesda consensus panel of microsatellite markers in the absence of proband normal tissue. BMC medical genetics. 2006; 7:5.

29. Elsaleh H, Iacopetta B. Microsatellite instability is a predictive marker for survival benefit from adjuvant chemotherapy in a population-based series of stage III colorectal carcinoma. Clinical colorectal cancer. 2001; 1:104-109.
30. Cao D, Leffert JJ, McCabe J, Kim B, Pizzorno G. Abnormalities in uridine homeostatic regulation and pyrimidine nucleotide metabolism as a consequence of the deletion of the uridine phosphorylase gene. The Journal of biological chemistry. 2005; 280:21169-21175.

31. Zu X, Yan R, Pan J, Zhong L, Cao Y, Ma J, Cai C, Huang D, Liu J, Chung FL, Liao DF, Cao D. Aldo-keto reductase $1 \mathrm{~B} 10$ protects human colon cells from DNA damage induced by electrophilic carbonyl compounds. Molecular carcinogenesis. 2016; 56:118-129.

32. Shen Y, Ma J, Yan R, Ling H, Li X, Yang W, Gao J, Huang C, Bu Y, Cao Y, He Y, Wan L, Zu X, et al. Impaired selfrenewal and increased colitis and dysplastic lesions in colonic mucosa of AKR1B8-deficient mice. Clinical cancer research. 2015; 21:1466-1476.

33. Gonzalez-Pons M, Cruz-Correa M. Colorectal Cancer Biomarkers: Where Are We Now? BioMed research international. 2015; 2015:149014.

34. Ahrendt SA, Decker PA, Doffek K, Wang B, Xu L, Demeure MJ, Jen J, Sidransky D. Microsatellite instability at selected tetranucleotide repeats is associated with p53 mutations in non-small cell lung cancer. Cancer research. 2000; 60:2488-2491.

35. Elsaleh H, Shannon B, Iacopetta B. Microsatellite instability as a molecular marker for very good survival in colorectal cancer patients receiving adjuvant chemotherapy. Gastroenterology. 2001; 120:1309-1310.

36. Samowitz WS, Curtin K, Ma KN, Schaffer D, Coleman LW, Leppert M, Slattery ML. Microsatellite instability in sporadic colon cancer is associated with an improved prognosis at the population level. Cancer epidemiology, biomarkers \& prevention. 2001; 10:917-923.

37. Brueckl WM, Heinze E, Milsmann C, Wein A, Koebnick C, Jung A, Croner RS, Brabletz T, Gunther K, Kirchner T, Hahn EG, Hohenberger W, Becker H, Reingruber B. Prognostic significance of microsatellite instability in curatively resected adenocarcinoma of the small intestine. Cancer letters. 2004; 203:181-190.

38. Sweetser S, Ahlquist DA. Multi-Target Stool DNA Test: Is the Future Here? Current gastroenterology reports. 2016; $18: 30$.

39. Kutzner N, Hoffmann I, Linke C, Thienel T, Grzegorczyk M, Urfer W, Martin D, Winde G, Traska T, Hohlbach G, Muller KM, Kuhnen C, Muller O. Non-invasive detection of colorectal tumours by the combined application of molecular diagnosis and the faecal occult blood test. Cancer letters. 2005; 229:33-41. 